International Journal of Applied Mathematics

Volume 33 No. $6 \quad 2020,1083-1098$

ISSN: 1311-1728 (printed version); ISSN: 1314-8060 (on-line version)

doi: http://dx.doi.org/10.12732/ijam.v33i6.9

\title{
ON FAITHFUL MATRIX REPRESENTATIONS OF $q$-DEFORMED LIE ALGEBRA FOR COUPLED QUANTIZED OSCILLATORS
}

\author{
L.A-M. Hanna \\ Dept. of Maths., Faculty of Science, Kuwait Univ. \\ P.O. Box 5969 Safat 13060 \\ Kuwait City - KUWAIT
}

\begin{abstract}
The polynomially deformed Lie algebra, $\mathfrak{l}_{q}:\left[K_{0}, K_{+}\right]_{q}=G\left(K_{+}\right)$, $\left[K_{-}, K_{0}\right]_{q}=G\left(K_{-}\right),\left[K_{+}, K_{-}\right]_{q}=P\left(K_{0}\right)$, is introduced as a generalized model of the coupled quantized oscillators model, where $G$ and $P$ are real polynomial functions, subject to the physical properties: $K_{0}$ is a real diagonal operator, and $K_{-}=K_{+}^{\dagger}$. Matrix representations are discussed and conditions are given for $G$ and $P$ to guarantee the existence of the faithful representations.
\end{abstract}

AMS Subject Classification: 17B10, 17B81

Key Words: matrix representations of Lie algebras

\section{Introduction}

Modeling of nonlinear quantum optical physics phenomena (like, multimode radiation fields on multiphoton processes mediums, many -body physics models [1], [2], investigating the statistics of particles that interpolate between Bosons and Fermions, [13]-[17], or study of $q$-deformed oscillators [12], [16]) is expressed as Hamiltonians whose non-linearities are of higher order than the quadratic forms. In turn, such highly nonlinear Hamiltonians are associated with nonlinear $q$-deformed Lie algebra or polynomially deformed Lie algebras $S U_{p d}(2)$, [3].

On the other hand, direct methods to solve for the evolution parameters or Schrödinger's wave equations of such nonlinear Hamiltonian models are tedious

Received: October 26, 2020

(C) 2020 Academic Publications 
in many cases . However, the use of Lie algebraic approach (e.g., [3] and references therein) in such nonlinear models has some advantages as compared with direct methods, (similar to the familiar Lie algebraic decomposition method for bilinear Hamiltonians, [21], [22]).

For such useful Lie algebraic decomposition methods, one requires faithful matrix representation for the generators of such deformed Lie algebra.

The intention of this paper is to consider the $q$-deformed algebra in a more general form that considers both types of deformation, namely, $q$-deformed Lie brackets and polynomially deformed Lie algebras, and investigate the faithful matrix representations of the generators of such deformed Lie algebras. Representations of degree 2 are the least possible degrees.

\section{Preliminaries}

Definition 1. Let $X$ and $Y$ be $n \times n$ matrices. The $q$-deformed Lie bracket of $X$ and $Y$ is defined as

$$
[X, Y]_{q}=X Y-q Y X \quad \text { for } \quad q \in \mathbb{R}
$$

For $q=0$, it is the ordinary matrix multiplication of $X$ and $Y$. Thus, throughout this paper, $q$ is supposed to be a nonzero real number. Whereas, for $q=1$, it is the ordinary Lie bracket. Thus, we always, write $[X, Y]_{1}$ as $[X, Y]$. In [11], e.g., $q$ was considered as $0 \leq q \leq 1$ for the model of Fermion Oscillators.

Faithful matrix representations of least degree of the Lie algebra $\mathfrak{l}$, were considered in [4]-[10], namely,

$$
\mathfrak{l}:\left[K_{0}, K_{ \pm}\right]= \pm r K_{ \pm} \text {and }\left[K_{+}, K_{-}\right]=P\left(K_{0}\right)
$$

where $P$ is a real polynomial function, subject to the physical properties, namely, $K_{0}$ is a real diagonal operator and $K_{-}=K_{+}^{\dagger}$ († is for Hermitian conjugation), with $K_{+}+K_{-}$is a real operator, in order to satisfy the Hermiticity of the Hamiltonian of the coupled quantized optical atoms, namely, [10]

$$
H=\omega K_{0}+\lambda(t)\left(K_{+}+K_{-}\right) .
$$

In this paper, we discuss, the faithful matrix representations for $\mathfrak{l}_{q}$, where

$$
\mathfrak{l}_{q}:\left[K_{0}, K_{+}\right]_{q}=G\left(K_{+}\right)
$$




$$
\begin{aligned}
& {\left[K_{-}, K_{0}\right]_{q}=G\left(K_{-}\right),} \\
& {\left[K_{+}, K_{-}\right]_{q}=P\left(K_{0}\right),}
\end{aligned}
$$

subject to the physical properties, namely, $K_{-}=K_{+}^{\dagger}$ and $K_{0}$ is a real diagonal operator with $K_{+}+K_{-}$is a real operator, in order to satisfy the Hermiticity of the Hamiltonian (2), where $P$ and are real polynomials.

In (3)-(5), $\mathfrak{l}_{q}$ is introduced as a more generalization of the models considered in [4]-[10]. It is the $q$-deformed and polynomially deformed model of $\mathfrak{l}$ in (1). As on setting $q=1$, then $\mathfrak{l}_{1}$ becomes the Lie algebra $\mathfrak{l}$, where $G(x)=r x$. So, for $q=1$ and with particular choices of $G$ and $P$, one gets the models in [4]-[9] and their special models, e.g., in [27], [28] and also, obtains their matrix representations.

\section{Basic properties of the $q$-deformed Lie Bracket for quantized Hamiltonians}

Some basic properties of the $q$-deformed Lie bracket are listed in the following theorem, whose proof can be driven immediately from the definition.

Theorem 2. Let $X, Y$ and $Z$ be $n \times n$ matrices, $q \in \mathbb{R}$ and $\alpha, \beta \in \mathbb{C}$. Then:

1. $\operatorname{tr}\left([X, Y]_{q}\right)=\operatorname{tr}\left([Y, X]_{q}\right)$.

2. $[X, Y]_{q}^{\dagger}=\left[Y^{\dagger}, X^{\dagger}\right]_{q}$.

3. $[X, Y]_{q}=q[X, Y]+(1-q) X Y$.

4. $[\alpha A, \beta B]_{q}=\alpha \beta[A, B]_{q}$.

5. $[X, Y]_{q}=[X, Y]+(1-q) Y X$.

6. $[X, X]_{q}=(1-q) X^{2}$.

7. $[X, Y+Z]_{q}=[X, Y]_{q}+[X, Z]_{q}$.

8. $[Y, X]_{q}=[X, Y]_{q}-(1+q)[X, Y]$.

9. $[X+Y, Z]_{q}=[X, Z]_{q}+[Y, Z]_{q}$.

10. $[X, Y]_{q}=(1+q)[X, Y]+[Y, X]_{q}$. 
11. $[X, Y]_{q}=(1+q)[X, Y]+[Y, X]_{q}$.

12. $[X, Y]_{q}=-q[Y, X]_{q}+\left(1-q^{2}\right) X Y$.

13. $[Y, X]_{q}=-q[X, Y]_{q}+\left(1-q^{2}\right) Y X$.

14. $[X, Y]_{q}-[Y, X]_{q}=(1+q)[X, Y]$.

15. $\left[[X, Y]_{q}, Z\right]_{q}=X Y Z-q Y X Z-q Z X Y-q^{2} Z Y X$.

16. $\left[X,[Y, Z]_{q}\right]_{q}=X Y Z-q Y Z X-q X Z Y+q^{2} Z Y X$.

17. $\left[[X, Y]_{q}, Z\right]_{q}-\left[X,[Y, Z]_{q}\right]_{q}=q[X, Z] Y+q Y[Z, X]$.

18. $\left[X,[Y, Z]_{q}\right]-\left[[X, Y]_{q}, Z\right]_{q}=q Y[X, Z]+q[Z, X] Y$.

19. $\left[X,[Y, Z]_{q}\right]_{q}+\left[Y,[Z, X]_{q}\right]_{q}+\left[Z,[X, Y]_{q}\right]_{q}$

$$
=(1-q)[(X Y Z+Y Z X+Z X Y)
$$

$-q(X Z Y+Y X Z+Z Y X)]$.

Proposition 3. The polynomial function $G(x)$ of $x$ satisfies

$$
\begin{aligned}
G\left(K_{-}\right) & =\left[K_{0}, K_{-}\right]_{q}-(1+q)\left[K_{0}, K_{-}\right] \\
& =-q\left[K_{0}, K_{-}\right]_{q}+\left(1-q^{2}\right) K_{-} K_{0}
\end{aligned}
$$

Proof. From (3), by using parts 2 and 10, of Theorem 2, we have

$$
\begin{aligned}
G\left(K_{-}\right)=\left(G\left(K_{+}\right)\right)^{\dagger}=\left[K_{0}, K_{+}\right]_{q}^{\dagger}=\left[K_{+\dagger}^{\dagger}, K_{0}^{\dagger}\right]_{q} & =\left[K_{-}, K_{0}\right]_{q} \\
& =\left[K_{0}, K_{-}\right]_{q}+(1+q)\left[K_{-}, K_{0}\right]
\end{aligned}
$$

and from part (12) of Theorem 2, we have

$$
\left[K_{-}, K_{0}\right]_{q}=-q\left[K_{0}, K_{-}\right]_{q}+\left(1-q^{2}\right) K_{-} K_{0}
$$


Theorem 4. The defining relations of $\mathfrak{l}_{q}$ can be either:

$$
\left[K_{0}, K_{+}\right]_{q}=G\left(K_{+}\right), \text {and }\left[K_{+}, K_{-}\right]_{q}=P\left(K_{0}\right)
$$

or

$$
\left[K_{-}, K_{0}\right]_{q}=G\left(K_{-}\right), \text {and }\left[K_{+}, K_{-}\right]_{q}=P\left(K_{0}\right),
$$

where $P$ and $G$ are real polynomials, subject to the physical properties, namely, $K_{-}=K_{+}^{\dagger}$ and $K_{0}$ is a real diagonal operator with $K_{+}+K_{-}$is a real operator.

\section{Faithful representations of $\mathfrak{l}_{q}$}

Unless otherwise stated, $A, B$ and $C$ are representation matrices for $K_{+}, K_{-}$ and $K_{0}$, respectively. All representations into consideration are supposed to satisfy the physical properties, namely, $B=A^{\dagger}$ and $C$ is a real diagonal matrix with $A+B$ is real a matrix. Also, $P(x)$ and $G(x)$ are polynomial functions in $\mathbb{R}[x]$.

The following lemma shows that, interchanging the position of two diagonal elements of $C$, leads to a conjugate representation of $\mathfrak{l}_{q}$ satisfying the physical requirements.

Lemma 5. Let $\delta=(u v)$ be a permutation in the symmetric group $S_{n}$. When applying $\delta$ to the rows as well as to the columns of the $n \times n$ matrices $A, B$ and $C$, we obtain a conjugate matrix representation of $\mathfrak{l}_{q}$ satisfying the physical requirements of degree $n$.

Proof. Let $E$ be the elementary matrix obtained by applying $\delta$ to the rows of $I_{n}$. Since $E=E^{-1}=E^{T}=E^{\dagger}$, then $A^{\prime}=E^{-1} A E, B^{\prime}=E^{-1} B E$ and $C^{\prime}=E^{-1} C E$ are representation matrices for $K_{+}, K_{-}$and $K_{0}$, respectively. For, $\left[C^{\prime}, A^{\prime}\right]_{q}=\left(E^{-1} C E\right)\left(E^{-1}\right.$

$A E)-q\left(E^{-1} A E\right)\left(E^{-1} C E\right)=E^{-1}(C A-q A C) E=E^{-1}[C, A]_{q} E$

$=E^{-1} G(A) E=G\left(E^{-1} A E\right)=G\left(A^{\prime}\right)$. Similarly, $\left[A^{\prime}, B^{\prime}\right]_{q}=P\left(C^{\prime}\right)$. The physical properties of $\mathfrak{l}_{q}$ are also satisfied in respective with the matrices $A^{\prime}, B^{\prime}$ and $C^{\prime}$.

So, we consider $C$ consists of $k$ diagonal blocks of scalar matrices corresponding to different scalars. That is $C=\operatorname{diag}\left(\delta_{1} I_{n_{1}}, \delta_{2} I_{n_{2}}, \ldots\right.$, 
$\left.\delta_{k} I_{n_{k}}\right)$, for different real numbers $\delta_{1}, \delta_{2}, \ldots, \delta_{k}$ with $\sum_{i=1}^{k} n_{i}=n$, where $n$ is the degree of the representation.

The following equations (7)-(9) are necessary relations for $A, B$ and $C$, which are obtained from $(3)$ and (5), respectively. For $i, j=1,2, \ldots, n$, we have

$$
(G(A))_{i j}=a_{i j}\left(c_{i i}-q c_{j j}\right)
$$

and

$$
\begin{aligned}
& \sum_{t=1}^{n}\left(a_{i t} \bar{a}_{j t}-q a_{t j} \bar{a}_{t i}\right)=\text { for } i \neq j \\
& \sum_{t=1}^{n}\left(\left|a_{i t}\right|^{2}-q\left|a_{t i}\right|^{2}\right)=P\left(c_{i i}\right) .
\end{aligned}
$$

\section{Faithful matrix representations of $\mathfrak{l}_{q}$ of degree 2 , where $F$ and $G$ are real linear polynomials}

Since $\mathfrak{l}_{q}$ is generated by 3 generators, namely, $K_{ \pm}$and $K_{0}$, then the least possible degree of a faithful matrix representation is 2 . In such a case, $A, B=A^{\dagger}$ and $C=$ $\operatorname{diag}\left(c_{1}, c_{2}\right)$ with $c_{1}, c_{2} \in \mathbb{R}$, are linearly independent $2 \times 2$ matrices satisfying the physical conditions. It should be noticed that when a contradiction occurs in a representation of degree 2 , when $c_{1} \neq c_{2}$, a contradiction is going to occur in representations of higher degrees between two different diagonal blocks of the representation matrix of $K_{0}$. Also, if a contradiction may occur when $c_{1}=c_{2}$, then it will occur in representations of higher degrees in the same diagonal block of the representation matrix of $K_{0}$. This is why representations of degree 2 are of particular importance. The $q$-deformed Lie algebra (3)-(5) is a generalization to the Lie algebras in [4] and [9] and many other models. This is why the case where $F$ and $G$ are linear polynomials is practically important.

Unless otherwise stated we consider, from now on, $P(x)=\mu x+\nu$ and $G(x)=\alpha x+\beta$ for real $\alpha, \beta, \mu, \nu$ and

$$
A=\left[\begin{array}{ll}
a & b \\
c & d
\end{array}\right], B=A^{\dagger} \text { and } C=\operatorname{diag}\left(c_{1}, c_{2}\right),
$$

where $c_{1}, c_{2} \in \mathbb{R}$. Thus, we have from (5) and (3), respectively, that $[A, B]_{q}=$ $\mu C+\nu I$

$=\left[\begin{array}{cc}(1-q)|a|^{2}+|b|^{2}-|c|^{2} q & (a \bar{c}+b \bar{d})-(b \bar{a}+d \bar{c}) q \\ \overline{(a \bar{c}+b \bar{d})-(b \bar{a}+d \bar{c}) q} & |c|^{2}-|b|^{2} q+(1-q)|d|^{2}\end{array}\right]$ 


$$
[C, A]_{q}=\left[\begin{array}{cc}
(1-q) a c_{1} & b\left(c_{1}-q c_{2}\right) \\
c\left(c_{2}-q c_{1}\right) & (1-q) d c_{2}
\end{array}\right]=\alpha A+\beta I .
$$

In the following theorem we state some basic and necessary properties of the representation matrices, enough to find and decide whether the Lie algebra $\mathfrak{l}_{q}$ has a faithful representation of degree 2 .

Theorem 6. Consider the representation matrices $A, B=A^{\dagger}$ and $C=$ $\operatorname{diag}\left(c_{1}, c_{2}\right)$ of $K_{+}, K_{-}$and $K_{0}$, respectively as in (10), and $P(C)=\mu C+\nu I$ and $G(A)=\alpha A+\beta I$. Then

1. $a$ and $d$ are real and $b \neq \bar{c}$.

2. $\alpha=\left(c_{1}-q c_{2}\right)$, if $b \neq 0$ or $\alpha=\left(c_{2}-q c_{1}\right)$ if $c \neq 0$.

If $b c \neq 0$, then either $q=-1$ or $C$ is a scalar matrix.

3. $\beta=\left[(1-q) c_{1}-\alpha\right] a=\left[(1-q) c_{2}-\alpha\right] d$ $=\frac{1}{2}\left[(1-q)\left(a c_{1}+d c_{2}\right)-\alpha(a+d)\right]$.

4. $\mu c_{1}+\nu=(1-q) a^{2}+\left(|b|^{2}-q|c|^{2}\right)$ and $\mu c_{2}+\nu=(1-q) d^{2}+\left(|c|^{2}-q|b|^{2}\right)$.

5. $\mu\left(c_{1}-c_{2}\right)=(1-q)\left(a^{2}-d^{2}\right)+(1+q)\left(|b|^{2}-|c|^{2}\right)$.

6. $b(d-q a)+\bar{c}(a-q d)=0$.

Moreover, if $A, B$ and $C$ are linearly independent, then the representation is faithful.

Proof. On comparing the diagonal elements on both sides of (12), we get, $a\left[(1-q) c_{1}-\alpha\right]=\beta=d\left[(1-q) c_{2}-\alpha\right]$. This shows that, $a$ and $d$ must be real numbers. If $b=\bar{c}$, then $A$ is Hermitian matrix and $B=A$ and the representation is not faithful. That proves parts 1 and 3 , which is completed by adding the first two equations of it. Similarly, on comparing the non-diagonal elements of on both sides of (12) and solving for $\alpha$ we prove part 2. If $b c \neq 0$, then $\left(c_{1}-c_{2}\right)(q+1)=0$. Thus, $c_{1}=c_{2}$ or $q=-1$. Parts $4-6$ are by comparing elements of matrices in both sides of the matrices $P(C)$ in (11). 
Theorem 7. If $\alpha \beta \neq 0$ and $b c \neq 0$, then $\mathfrak{l}_{q}$ has no faithful real representation of degree 2 .

Proof. The proof depends on Theorem 6. So, from part 3 of Theorem 6 as $b c \neq 0$, then either $q=-1$ or $c_{1}=c_{2}=r \in \mathbb{R}$.

If $q=-1$, then from part 2, $\alpha=c_{1}+c_{2}$ and from part 3, we have, $(a+d)\left(c_{1}-c_{2}\right)=0$.

Case 1: $a=-d$. Thus, from part $5, \mu\left(c_{1}-c_{2}\right)=0$. If $\mu=0$, then $P(C)=$ $\nu I$, which leads to $c_{1}=c_{2}$.

Case 2: $c_{1}=c_{2}=r$. Thus, from part 2, as $q=-1$, then $\alpha=2 r$, and hence, from part 3 , we get $\beta=0$, which is a contradiction.

On the other hand if $q \neq-1$ and $C=r I$, a scalar matrix, then from part $2, \alpha=(1-q) r$ and from part $3 \beta=[(1-q) r-\alpha] a=0$, contradicting $\beta \neq 0$.

It should be pointed out that if $b=c=0$, then the representation is not faithful. Also, as results of parts 2 and 3 of Theorem 6 , if $C=r I$, a scalar matrix and $b \neq 0$ and $c=0$, then $\beta=0$. Representation matrices obtained, when $b=0$ and $c \neq 0$ are similar to those when $b \neq 0$ and $c=0$, by changing $B$ and $A$.

So, we always discuss representations with $c=0$ and $b \neq 0$.

In the following theorem we introduce conditions and a procedure to calculate $A$ and $C$ to guarantee a faithful matrix representations for $\mathfrak{l}_{q}$.

Theorem 8. Let $q^{2} \neq 1$ and $\alpha \neq 0$. A necessary and sufficient condition for $\mathfrak{l}_{q}$ to have a faithful representation with $c=0$ and $a b \neq 0$, is that $a$ is a real root of equation (13), satisfying that $|b|^{2}>0$, where the elements of these representation matrices are given by the following sequence of equations:

1. $a$ is calculated as a real root of the following equation satisfying that $|b|^{2}>0$,

2. $a^{3}\left(-q^{2}+q^{3}+q^{4}-q^{5}\right)+a\left(q \nu-q^{3} \nu+q \alpha \mu+q^{2} \alpha \mu\right)$ $+\beta \mu\left(1+q^{2}\right)=0$,

3. $|b|^{2}=\frac{a^{3}\left(-q+2 q^{2}-2 q^{3}+2 q^{4}-q^{5}\right)+2 a\left(q \alpha \mu+q \nu-q^{2} \nu\right)+\beta \mu(1+q)}{a q(q-1)^{2}}$,

4. $d=a q$,

5. $c_{1}=\frac{\beta+a \alpha}{a(1-q)}$ and $c_{2}=\frac{\beta+\alpha a q}{a q(1-q)}$. 
Proof. This proof is directly driven from Theorem 6. So, from part 6, $d=a q$. And from part $3, c_{2}=\frac{\beta+\alpha a q}{a q(1-q)}$, and substitution in part 3, we get $c_{1}=$ $\frac{\beta+a \alpha}{a(1-q)}$. And from part 4 , we get, by addition, $(1-q)\left(1+q^{2}\right) a^{2}+(1-q)|b|^{2}=$ $\mu\left(\frac{\beta+a \alpha}{a(1-q)}+\frac{\beta+\alpha a q}{a q(1-q)}\right)+2 \nu$. Thus,

$$
(1-q)|b|^{2}=\mu\left(\frac{\beta+a \alpha}{a(1-q)}+\frac{\beta+\alpha a q}{a q(1-q)}\right)+2 \nu-(1-q)\left(1+q^{2}\right) a^{2} .
$$

Therefore, $|b|^{2}=\frac{\mu\left(\frac{\beta+a \alpha}{a(1-q)}+\frac{\beta+\alpha q q}{a q(1-q)}\right)+2 \nu-(1-q)\left(1+q^{2}\right) a^{2}}{1-q}$, which must be positive and after some reductions, we prove (14). From part $4 \mu\left(\frac{\beta+a \alpha}{a(1-q)}\right)+\nu=(1-q) a^{2}+$ $|b|^{2}$. Thus, $\mu\left(\frac{\beta+a \alpha}{a(1-q)}\right)+\nu-(1-q) a^{2}-|b|^{2}=0$. On using (14), we have

$$
\begin{aligned}
& \mu\left(\frac{\beta+a \alpha}{a(1-q)}\right)+\nu-(1-q) a^{2} \\
& -\frac{a^{3}\left(-q+2 q^{2}-2 q^{3}+2 q^{4}-q^{5}\right)+2 a\left(q \alpha \mu+q \nu-q^{2} \nu\right)+\beta \mu(1+q)}{a q(q-1)^{2}}=0 .
\end{aligned}
$$

Therefore, $\frac{\left(\beta \mu-a^{3} q^{2}+a^{3} q^{3}+a^{3} q^{4}-a^{3} q^{5}+a q \nu-a q^{3} \nu+q^{2} \beta \mu+a q \alpha \mu+a q^{2} \alpha \mu\right)}{a q(q-1)^{2}}=0$. Which yields to $\beta \mu-a^{3} q^{2}+a^{3} q^{3}+a^{3} q^{4}-a^{3} q^{5}+a q \nu-a q^{3} \nu+q^{2} \beta \mu+a q \alpha \mu+a q^{2} \alpha \mu=0$, and so is (13). Therefore, $\mathfrak{l}_{q}$ has a faithful representation if and only if, $a$ is calculated as a real root of equation (13) satisfying equation (14), with $|b|^{2}>0$.

The following examples are to illustrate the above procedure.

Example 9. Given that $q=\frac{1}{2}, \alpha=1, \beta=-\frac{31}{40}, \mu=3$, and $\nu=2$.

Substitute in (13), we have, $a^{3}-32 a+31=0$, then $(a-1)\left(a^{2}+a-31\right)=0$. The roots of this equation are: $1, \frac{5}{2} \sqrt{5}-\frac{1}{2}$ and $-\frac{5}{2} \sqrt{5}-\frac{1}{2}$. But $|b|^{2}$ is only positive for $a=1$, thus $a=1$. And since from (14) and assuming that the representation is real, we have $b^{2}=\frac{57}{20}$. So, take $b= \pm \sqrt{\frac{57}{20}}, c=0, d=\frac{1}{2}$, $c_{1}=\frac{9}{20}$ and $c_{2}=-\frac{11}{10}$.

Example 10. Given that $q=2, \alpha=2, \beta=2, \mu=3, \nu=-1$.

We have from (13), the equation $2 a^{3}-7 a-5=0$ has roots: $\frac{1+\sqrt{11}}{2}, \frac{1-\sqrt{11}}{2}$ and -1 . But $|b|^{2}<0$ for $\frac{1+\sqrt{11}}{2}$ and $\frac{1-\sqrt{11}}{2}$, rejected. So, $a=-1$. Thus, $d=-2$. And 
from (14), we have $|b|^{2}=0$ and $c_{1}=0, c_{2}=-1$. Thus, $A=B=\operatorname{diag}(-1,-2)$ and $C=\operatorname{diag}(0,-1)$. Which gives a representation that is not faithful.

\section{6. $G(x)$ is a polynomial of zero absolute term}

Practically, as in [10], a particular interest is given for representations where $A$ is a nilpotent matrix or a triangular matrix of zero diagonal elements. This is when $\beta=0$ as in the following theorem, where $G$ is considered as a linear polynomial.

Theorem 11. Let $q \neq-1$ and $G(x)=\alpha x$, where $\alpha \neq 0$. Then the representation of $\mathfrak{l}_{q}$ is conjugate to a representation where $A$ is $n \times n$ real upper (or lower) triangular matrix with zero diagonal entries. Furthermore, if $A+B$ is real matrix then the representation is real.

Proof. From (3), (4), we have for $i, j=1, \ldots, n$,

$$
\alpha a_{i j}=\sum_{t=1}^{n}\left(\delta_{i t} c_{i t} a_{t j}-q a_{i t} \delta_{t j} c_{t j}\right),
$$

i.e.,

$$
a_{i j}\left(c_{i i}-q c_{j j}-\alpha\right)=0
$$

and

$$
a_{j i}\left(c_{j j}-q c_{i i}-\alpha\right)=0 .
$$

Suppose, $a_{i j} a_{j i} \neq 0$ for $i \neq j$, we have

$$
\begin{aligned}
& c_{i i}-q c_{j j}=\alpha, \\
& c_{j j}-q c_{i i}=\alpha .
\end{aligned}
$$

By subtraction in (15), (16), we get $(1+q)\left(c_{i i}-c_{j j}\right)=0$. Since $q \neq-1$, then for two different diagonal blocks in $C$, where, $c_{i i} \neq c_{j j}$, we have that $a_{i j}$ and $a_{j i}$ cannot both be nonzero. Thus, one can arrange the matrix $C$, which is equivalent to getting a conjugate representation, in order to obtain $A$ as an upper (or lower) triangular matrix.

Now, consider representation of degree 2, where $A, B$ and $C$ are as in (10). If $\beta=0, c=0$ and $b \neq 0$, then from parts 2 and 3 of Theorem 6 , we get $a q\left(c_{2}-c_{1}\right)=0$, and $d\left(c_{2}-c_{1}\right)=0$. Then, $a=0$ and $d=0$, when $C$ is not a scalar matrix. And we get, $A$ as a nilpotent matrix of degree of nilpotency 2 and 
$G(0)=0$. This allows to consider $G$ of a degree greater than 1 . In the following theorem, the necessary and sufficient conditions are exhibited to have faithful representation for $\mathfrak{l}_{q}$, and formulae are given to calculate the representation matrices, even when $P(x)$ is not linear and $G(x)=\alpha x+\sum_{i \geq 2} \alpha_{i} x^{i}$ and $A$ as a nilpotent matrix.

Theorem 12. If $G(A)=\alpha A+\sum_{i \geq 2} \alpha_{i} A^{i}$, where the matrices $A=$ $\left[\begin{array}{ll}0 & b \\ 0 & 0\end{array}\right], B=A^{\dagger}$ and $C=\operatorname{diag}\left(c_{1}, c_{2}\right)$ are representation matrices of $K_{+}, K_{-}$ and $K_{0}$, respectively, then $\mathfrak{l}_{q}$ has a faithful representation if and only if there exists a real numbers $c_{1}$ such that $P\left(c_{1}\right)>0$. In this case, $b= \pm \sqrt{P\left(c_{1}\right)}$ and $c_{2}=\frac{c_{1}-\alpha}{q}$.

Proof. Since $b \neq 0$, then from part 1 of Theorem $6, c_{2}=\frac{c_{1}-\alpha}{q}$. From (11), as $a=c=d=0$, we have $P\left(c_{1}\right)=\left([A, B]_{q}\right)_{11}=|b|^{2}>0$, for a faithful representation. Thus, $b=\sqrt{P\left(c_{1}\right)}$.

Actually, From (11), $\operatorname{tr}\left([A, B]_{q}\right)=P\left(c_{1}\right)+P\left(c_{2}\right)=-|b|^{2}(q-1)=$ $-P\left(c_{1}\right)(q-1)$. Therefore,

$$
P\left(c_{2}\right)+q P\left(c_{1}\right)=0 .
$$

Example 13. Let $q=-1, P(x)=x^{2}-2 x-3$ and $G(x)=2 x+11 x^{4}$. On choosing, $c_{1}=4$, since $P(4)=5>0$. Then $c_{2}=\frac{4-2}{-1}=-2$. Then $A=$ $\left[\begin{array}{rr}0 & \sqrt{5} \\ 0 & 0\end{array}\right], B=A^{\dagger}$, and $C=\operatorname{diag}(4,-2)$ are the representation matrices of $\mathfrak{l}_{-1}:\left[K_{0}, K_{+}\right]_{-1}=2 K_{+}+11 K_{+}^{4}$, and $\left[K_{+}, K_{-}\right]_{-1}=K_{0}^{2}-2 K_{0}-3 K_{0}^{0}$.

While on choosing, $c_{1}=5$, since $P(5)=12>0$. Then $c_{2}=\frac{5-2}{-1}=-3$ and $P\left(c_{2}\right)=12$. Then $A=\left[\begin{array}{rr}0 & \sqrt{12} \\ 0 & 0\end{array}\right], B=A^{\dagger}$, and $C=\operatorname{diag}(4,-2)$ are another representation matrices of $\mathfrak{l}_{-1}$.

Example 14. If $q=\frac{1}{3}, P(x)=2 x^{2}-3 x-\frac{11}{4}$ and $G(x)=3 x^{4}-5 x^{3}+$ $3 x^{2}-\frac{5}{3} x$. Choose, $c_{1}=-1$, since $P(-1)=\frac{9}{4}>0$. Then $c_{2}=2$. Then $A=$ $\left[\begin{array}{cc}0 & \frac{3}{2} \\ 0 & 0\end{array}\right], B=A^{\dagger}$ and $C=\operatorname{diag}(-1,2)$ are representation matrices of a faithful representation of $\mathfrak{l}_{1 / 3}:\left[K_{0}, K_{+}\right]_{1 / 3}=G\left(K_{+}\right)$, and $\left[K_{+}, K_{-}\right]_{1 / 3}=P\left(K_{0}\right)$. 
Example 15. If $q=1, P(x)=-x^{2}+2 x$ and $G(x)=x^{2}$. Choose, $c_{1}=1$ since $P(1)=1>0$. Then $c_{2}=\frac{1-0}{2}=\frac{1}{2}$. Then $A=\left[\begin{array}{ll}0 & 1 \\ 0 & 0\end{array}\right], B=A^{\dagger}, C=$ $\operatorname{diag}\left(1, \frac{1}{2}\right)$.

\section{Generalized coupling quantized oscillators model}

We conclude this paper by introducing a generalized model for the coupling oscillators model. and considering its faithful representations of least degree of the $q$-deformed Lie algebra $\mathfrak{l}_{q}$, which is of a practically important case. This case in a generalization of the $q$-deformed $s l_{p d}(2, \mathbb{R}),[26]$. Also, in [9], it was considered that $q=1$, while $G\left(K_{+}\right)=\alpha K_{+}$. While it was considered in [5], [7], that $q=1, G\left(K_{0}\right)=\alpha K_{+}$and $P\left(K_{0}\right)=\mu K_{0}$.

Theorem 16. The $q$-deformed lie algebra generated by $K_{+}, K_{-}=K_{+}^{\dagger}$, and real diagonal operator $K_{0}$, satisfying

$$
\begin{aligned}
& \mathfrak{l}_{q}: {\left[K_{0}, K_{+}\right]_{q}=\alpha K_{+}, } \\
& {\left[K_{-}, K_{0}\right]_{q}=\alpha K_{-}, } \\
& {\left[K_{+}, K_{-}\right]_{q}=\mu K_{0}, \text { where } \alpha \mu>0, }
\end{aligned}
$$

and $K_{+}+K_{-}$is real operator, has real faithful representations of degree 2 as least degree, whose representation matrices $A, B$ and $C$ for $K_{+}, K_{-}$and $K_{0}$, respectively, where,

1. $A=\left[\begin{array}{rr}0 & \sqrt{\frac{\alpha \mu}{q^{2}+1}} \\ 0 & 0\end{array}\right], B=A_{+}^{\dagger}$ and $C=\left[\begin{array}{rr}\frac{\alpha}{q^{2}+1} & 0 \\ 0 & -\frac{\alpha q}{q^{2}+1}\end{array}\right]$,

2. $A=\left[\begin{array}{rr}0 & -\sqrt{\frac{\alpha \mu}{q^{2}+1}} \\ 0 & 0\end{array}\right], B=A_{+}^{\dagger}$ and $C=\left[\begin{array}{rr}\frac{\alpha}{q^{2}+1} & 0 \\ 0 & -\frac{\alpha q}{q^{2}+1}\end{array}\right]$,

3. $A=\left[\begin{array}{cc}0 & 0 \\ \sqrt{\frac{\alpha \mu}{q^{2}+1}} & 0\end{array}\right], B=A_{+}^{\dagger}$ and $C=\left[\begin{array}{rr}-\frac{\alpha q}{q^{2}+1} & 0 \\ 0 & \frac{\alpha}{q^{2}+1}\end{array}\right]$, and

4. $A=\left[\begin{array}{cc}0 & 0 \\ -\sqrt{\frac{\alpha \mu}{q^{2}+1}} & 0\end{array}\right], B=A_{+}^{\dagger}$ and $C=\left[\begin{array}{rr}-\frac{\alpha q}{q^{2}+1} & 0 \\ 0 & \frac{\alpha}{q^{2}+1}\end{array}\right]$. 
Proof. Since $P\left(c_{1}\right)=\mu c_{1}=|b|^{2}$ and $P\left(c_{2}\right)=\mu c_{2}=\mu\left(\frac{c_{1}-\alpha}{q}\right)$, from Theorem 10. Using (17), we have $\mu\left(\frac{c_{1}-\alpha}{q}\right)+q\left(\mu c_{1}\right)=0$. Then $\mu\left[c_{1}\left(q^{2}+1\right)-\alpha\right]=$ 0 . Thus we have, $c_{1}=\frac{\alpha}{q^{2}+1}$ and hence, $c_{2}=-\frac{\alpha q}{q^{2}+1}$ and also, $|b|^{2}=\frac{\alpha \mu}{q^{2}+1}$.

\section{Summary}

The $q$-deformed Lie algebra $\mathfrak{l}_{q}$, is a generalization of deformed Lie algebras for quantized Hamiltonians. It was shown that $\mathfrak{l}_{q}$ has two types of faithful matrix representations. If the absolute term of the polynomial $G\left(K_{+}\right)$is zero, then the matrices representing $K_{+}$and $K_{-}$are nilpotent matrices of degree 2 as least degree. The formulae to compute the representation matrices are given together with the conditions to guarantee that these matrices satisfy the physical properties and the representation is faithful. If the absolute term of $G\left(K_{+}\right)$is not zero, then the representation matrix of $K_{+}$is a triangular matrix. The conditions and formulae are given to evaluate the faithful representations for linear polynomials $P\left(K_{0}\right)$ and $G\left(K_{+}\right)$. So,

(1) If $G\left(K_{+}\right)=\alpha K_{+}+\beta, \beta \neq 0$, then $\mathfrak{l}_{q}$, has faithful representation of degree 2 as least degree, if and only if the upper triangular matrix $A=\left[a_{i j}\right]$ representing $K_{+}$, is such that: $a_{11}$ is a real root of equation (13), satisfying that $|b|^{2}$ is positive form (14), where $q \neq 1$.

(2) If $G\left(K_{+}\right)=\alpha K_{+}+\sum_{\ell>1} \alpha_{\ell} K_{+}^{\ell}$ then the representation matrix of $K_{+}$ is nilpotent matrices if and only if $\alpha=c_{1}-q c_{2}$ for some $c_{1}, c_{2} \in \mathbb{R}$ such that $P\left(c_{1}\right)>0$.

(3) The $q$-deformed Lie algebra $\mathfrak{l}_{q}$, was introduced as a generalized coupling oscillators model, that is when $P\left(K_{0}\right)=\mu K_{0}$ and $G\left(K_{+}\right)=\alpha K_{+}$. It was found that $\mathfrak{l}_{q}$ has faithful representation if and only if $\alpha \mu>0$.

Acknowledgements. The author is grateful to Prof. S.S. Hassan of Bahrain University for suggesting the problem and fruitful discussions. Thanks are due to Kuwait University.

\section{References}

[1] V.P. Karassiov, New Lie-algebraic structures in nonlinear problems of quantum optics and laser physics, J. of Soviet Laser Research, 13 (1992), 188-195. 
[2] V.P. Karassiov, $G$-invariant polynomial extensions of Lie algebras in quantum many-body physics, J. Phys. A: Math. Gen., 27 (1994), 153-165.

[3] V.P. Karassiov and A.B. Klimov, An algebraic approach to solving evolution problems in some nonlinear quantum models, Phys. Lett. A, 189 (1994), 43-51.

[4] A.B. Klimov and J.L. Romero, An algebraic solution of Lindblad-type master equation, J. Opt. B: Quantum Semiclass. Opt., 5 (2003), S316S321.

[5] L. A-M. Hanna, M.E. Khalifa and S.S. Hassan, On representations of Lie algebras for quantized Hamiltonians, Linear Algebra Appl., 266 (1997), 69-79.

[6] L. A-M. Hanna, On the classification of the Lie algebras, Linear Algebra Appl., 370 (2003), 251-256.

[7] L. A-M. Hanna, On matrix representations of deformed Lie algebras for quantized Hamiltonians, Linear Algebra Appl., 434 (2011), 507-513.

[8] L. A-M. Hanna, A deformed Tavis-Cummings model and its matrix representation, JP J. of Algebra, Number Theory and Applications, 35 (2014), 49-65.

[9] L. A-M. Hanna and S.S. Hassan, On matrix representations of deformed Lie algebras, Int. J. Theol. Phys., Group Th. and Nonlinear Opt., 13 (2010), 137-143.

[10] L. Hanna, R. Alharbey, S. Abdalla and S. Hassan, Algebraic method of solution of Schrödinger's equation of a quantum model, WSEAS Trans. on Mathematics, 19 (2020), 421-429.

[11] A. Algin, A comparative study on $q$-deformed Fermion oscillators, Int. J. Theor. Phys., 50 (2011), 1554-1568.

[12] P. Narayana Swamy, Interpolating statistics and $q$-deformed oscillator algebras, Intern. J. of Modern Physics, B, 20 (2006), 697-713.

[13] P. Narayana Swamy, q-deformed Fermions, Eur. Phys. J., B, 50 (2006), 291-294.

[14] P. Narayana Swamy, $q$-deformed Fermions: Algebra, Fock space and thermodynamics, Inten. J. of Modern Physics, B 20 (2006), 2537-2550. 
[15] S. Dey and V. Hussin, Noncommutative $q$-photon-added coherent states, Phys. Rev. A, 93 (2016), Art. 053824.

[16] S. Sargolzaeipor, H. Hassanabadi, W.S. Chung and A.N. Ikot, $q$-deformed oscillator algebra in fermionic and bosonic limits, Indian Acad. Sci., Pramana - J. Phys., 93 (2019), Art. 68.

[17] W.S. Chung and H. Hassanabadi, q-deformed quantum mechanics based on the $q$-addition, In: Advanced Science News, Fortschr. Phys. \# 1800111, Wiley-VCH Verlag GmbH \& Co. KGaA, Weinheim (2019).

[18] R.J.C. Spreeuw and J.P. Woerdman, Optical atoms, Progress in Optics, 31 (1993), 263-319.

[19] C.S. Adams, M. Sigel and Mlynek, Atom optics, Physics Reports, 240 (1994), Art. 143.

[20] W. Witschel, Ordered operator expansions by comparison, J. Phys. A, 8 (1975), 143-155.

[21] S. Steinberg, Applications of the Lie algebraic formulas of Baker, Campbell, Hausdorff, and Zassenhaus to the calculation of explicit solutions of partial differential equations, J. Differential Equations, 26 (1997), 404-434.

[22] S. Steinberg, Lie series, Lie transformations, and their applications, In: Lie Methods in Optics (J.S. Mondragón and K.B. Wolf, Eds., León, 1985), Lecture Notes in Phys., 250 (1986), 45-103.

[23] G. Dattoli, J.C. Gallardo and A. Torre, An algebraic view to the operatorial ordering and its applications to optics, La Rivisita del Nuovo Cimento, 11 (1988), Art. 1.

[24] G. Dattoli, P. Di Lazzaro, A. Torre, A spinor approach to the propagation in self-focusing fibers. Nuovo Cim. B, 105 (1990), 165-178.

[25] G. Dattoli, M. Richetta, G. Schettini and A. Torre, Lie algebraic methods and solutions of linear partial differential equations, J. Math. Phys., 31 (1990), Art. 2856.

[26] P.W. Higgs, Dynamical symmetries in a spherical geometry, I, J. Phys. A, 12 (1979), 309-323.

[27] M.A. Al-Gwaiz, M.S. Abdalla and S. Deshmuckh, Lie algebraic approach to the coupled-mode oscillator, J. Phys. A, 27 (1994), Art. 1275. 
[28] M. Sebawe Abdalla, H. Eleuch, T. Barakat, Exact analytical solutions of the wave function for some $q$-deformed potentials, Reports on Math. Phys., 71 (2013), 217-229. 